\title{
Software Practitioners Challenges in the Requirement Engineering Phase of Software Development
}

\author{
Abdool Qaiyum Mohabuth
}

\begin{abstract}
Software practitioners have always stressed upon the importance of having a dedicated formal Requirement Engineering (RE) stage in software development. Research has found that RE stage is among those factors that contribute towards project success. Decomposition of this stage in terms of elicitation, analysis, modeling and specification are well defined, but yet software developers claim they face issues as regards to the establishment of proper structural components during the RE stage. Project failures have often been associated with poor requirement definition where the practitioners approach to problem arising during RE phase has been criticised. Ensuring proper interaction among team members and maintaining good communication channel with clients have always been enlisted of being of prime importance in promoting project success in the RE phase. However, findings were mainly based on conventional wisdom. There is still lack of empirical evidence in terms of the identification of other factors that influenced the smooth running of the RE phase. This study aims at identifying the problems faced by practitioners in the RE phase through empirical evidence. An analysis of the influential factors with respect to the criticality for project successes is made. Assessment of the relevance of these factors according to length of projects and size of organisations are also made. A survey questionnaire was designed for identifying the problems faced by practitioners in the RE phase and their frequency of occurrences according to length of project were assessed. The questionnaire was administered to software developers at small, medium and large software enterprises. Semistructured interviews were also conducted with practitioners who have long years of experience as project leaders. Findings reveal that besides communication flaws there are other influential factors that hinder successful completion of the RE phase. 'Ti me boxing', 'Insufficient support from customer' and 'from own management', 'Gray areas in requirements' are among the hindering factors that make the RE phase vulnerable. Besides, it has been observed that there is uniformity across organisations. Small, medium and large development firms face similar issues as regards to the factors that affect the RE phase.
\end{abstract}

Keywords - communication, requirement, specification, practitioners, Time Boxing.

\section{INTRODUCTION}

RE represents the first phase of software development and is characterised by defining, documenting and maintaining requirements. It focuses on assessing if the system is useful to

Abdool Qaiyum Mohabuth, Senior Lecturer, University of Mauritius, Reduit, Mauritius the business (feasible study), discovering requirements (elicitation and analysis), converting these requirements into some standard format (specification), and checking that the requirements define the system that the customer wants (validation). RE should be "unambiguous", "consistent", "complete", "feasible", "traceable" and "verifiable" [1]. The tasks of software practitioners are in fact very challenging in achieving the standard set. It requires a vast number of viewpoints, roles, responsibilities and objectives as input to the system. Identified requirements must be clearly stated. Software practitioner uses a systematic approach to collect information from different sources and implement them into software processes. RE includes requirement development and requirement management. Development refers to tasks which are related to discovering, analysing, documenting and validating needs whereas requirement management focuses on traceability and change management tasks. [2] claimed that RE is one of the most important tools for gathering requirements, which is concerned with analysing and documenting the requirements.Misidentification of requirements is classified as one of the major source of client's dissatisfaction with the product delivered. For many practitioners of large and complex software systems, requirements are their biggest software engineering problem. [3] argued that the challenges for $\mathrm{RE}$ is communicating among people and managing complexity. There is adequate literature on the processes to follow to complete an appropriate RE, but there exist a dearth of literature on the factors hindering having a successful RE. [4] claimed that the state of empirical evidence in RE is particularly weak and dominated by isolated case studies andsmall-scale studies investigating aspects that cannot be generalised. In most cases, accurate evaluations starve in the future work section of publications [5].

\section{LITERATURE REVIEW}

$\mathrm{RE}$ is found to be amandatory stage for the success of every software project. It is said that RE is in fact a very challenging discipline in software development in order to make a software successful [6]. [7] classified the activities of RE as being associated with stakeholders' identification, requirements elicitation, prioritization and then implementation of the elicited requirements. This is further confirmed by [8] who stated that the RE process focuses on requirements elicitation 
and the representation of key business value. [9] stated that the success of software systems is directly associated with a set of key FunctionalRequirements and Non Function Requirements. If a software application or system meets the basic requirements criteria then, it is said that the software project is successful and vice versa. The key set of the requirements can only be obtained after applying key RE practices during different RE processes. [10] reported that $48 \%$ of development problems stem from problems with the requirements. [11] stated that solving problems related to requirement during the RE stage avoids high cost of rework in later states. [12] confirmed that if the requirement engineering phase went wrong, there is increasing risk that practitioners may build an entirely different product instead of the actual desired one.Besides, since RE involves a lot of interaction with stakeholders, practitioners have to establish proper communication channel with clients and have to maintain constant interaction with development team. Precise and consistent requirements directly contribute to appropriateness and cost effectiveness in the development of a system [13]; [14]. [3] focused on the communication aspects in REand found that RE isabout people communicating with people. In fact communication difficulties during RE, lack ofappropriate knowledge and assumption of understanding resulted into wrongful technology application design and development. This created havoc as from a business point of view, lack of understanding about system requirements always results in poor optimum design. System features might be unresponsive to the business needs. Quality of the process outcome is compromised due to failure in creating appropriate requirements documents. This impacted on the stakeholders' expectations. Therefore [12] stressed that one of the challenges for practitioners is to capable of preparing the requirements as detailed enough to be well understood without over constraining the system and predefining a whole host of things that may be better left to others downstream in the process to handle. The goal is to ensure that investment in requirement provides the right amountof specificity, leaving ambiguities aside.

\section{RESEARCH METHODOLOGY}

Four experienced software practitioners were interviewed to extract data about the main sources of problems that software developers encountered during the practice of the RE phase. A list of the most probable RE problem sources was identified and validated by the software practitioners. Survey research was found to be the most suitable method after extraction of the first set of data. A list of RE problems encountered were derived. Following which, a set of questionnaires were developed which was administered to a set of software practitionersinvolving the small, medium and large size software houses.The questionnaire contained section that included Likert scale questions to rate the degree of problem faced with a rating scale 1-5 (1: Insignificant Difficulties (ID), 2: Few Difficulties (FD), 3:Neutral (N), 4: Some Difficulties
(SD), 5: Notable Difficulties (ND). These were rated according to length of projects classified as 'short-term' and 'long term'. The extent at which these difficultiesaffected completion time of the RE phase were also measured by rating scale 1-5 (1: Not at all, 2: Alittle, 3: Neutral, 4: Much, 5: Very Much). Open-ended questions were also provided for enabling respondents to share their experiences and insights about problems faced in the RE phase. The questionnaire was stratified over the three types of software practitioners firms Small (S), Medium (M) and Large (L) for ensuring a good representation of practitioners across the different organizations. The size were determined by referring to Fernandez and Wagner (2015) who grouped organisations as small, medium, and large-sized based on the number of employees. Firms with up to 50 employees were considered small-sized, 51 to 250 were considered medium-sized organisations, and with more than 250 were considered largesized. Out of the 45 firms that completed the survey, 38 provided their number of employees.

\section{RESULTS AND DISCUSSION}

45 software practitioners responded to the survey and the data collected was quantified by using the SPSS software. $87 \%$ software practitioners confirmed the importance of the RE phase and this consolidates previous research conducted by [2];[15];[4]. The respondents agreed that ignorance of the features of RE stage lead to subsequent hassle in subsequent development phase. $77 \%$ of the practitioners stressed that planning of the RE phase should be discussed both with team members as well as clients. There are some similarities between both types of project which means that practitioners may face almost same level of difficulty on short or long term projects. Table 1 shows the mean rank of the degree of difficulties experienced by practitioners on short and long term project. Leaving gray areas in requirements is seen to be the most complex factor identified in both short and long term project (mean $4.62 \& 4.58$ ). This presents a great challenge to practitioners in clearing out the uncertainties. Other factors such as 'Inadequate customer support', 'Communication flaws', 'Time-Boxing', 'Gold Plating' and 'Inadequate senior mgt support' have been more highly rated (mean $>4$ ) than 'No formal defined responsibilities', 'Weak access to customer needs', 'Priority Shift' (mean $<4$ ), while 'Terminological problem', 'Immeasurable non functional requirements'and 'Inappropriate supporting tools' and 'Lack of technical expertise' are not very prevalent (mean <3). Responses regarding the causes of difficulties about leaving gray areas in the RE phase were predominantly related to deficiencies in having a coherent structure in following up and clearing out issues with the customers which resulted in incompleteness $(23 \%)$, impression (12\%), misunderstanding (32\%), inferior problem assessment (15\%) and unclear goals (18\%). Other significant causes of difficulties were related to the lack of IT knowledge and resistance to change on behalf of customers.'Gray Areas', 'Inadequate Support', 
'Communication flaws', 'Time-Boxing', 'Gold Plating' are those items that may be classified as being the most hindering factors for having successful REs, since they have been found to carry high average rating, more than $50 \%$ of the respondents identify these as being difficult and notablydifficult.No formal defined responsibilities', 'Weak access to customer needs', 'Priority Shift' may be classified as being of moderate difficulty in the RE phase as their level of difficulty has been found to be average. The other factors such as Inappropriate supporting tools' and 'Lack of technical expertise' are of low degree of difficulty in the RE phase and therefore they may carry less priority. However, respondents do not agree that these factors should be discarded as they do contribute towards successful REs.

TABLE I: DEGREE OF DIFFICULTY EXPERIENCED IN RE PHASE

\begin{tabular}{|l|r|r|r|c|}
\hline Factors & $\begin{array}{c}\text { Mean } \\
\text { Rank } \\
\text { Short } \\
\text { Term } \\
\text { Project }\end{array}$ & $\begin{array}{c}\text { SD } \\
\text { Short } \\
\text { Project }\end{array}$ & $\begin{array}{c}\text { Mean } \\
\text { Rank } \\
\text { Long } \\
\text { Term } \\
\text { Project }\end{array}$ & $\begin{array}{c}\text { SD } \\
\text { Project }\end{array}$ \\
\hline $\begin{array}{l}\text { Gray areas in } \\
\text { requirements }\end{array}$ & 4.62 & 0.576 & 4.58 & $\begin{array}{c}0.06 \\
9\end{array}$ \\
\hline $\begin{array}{l}\text { No formal } \\
\text { defined } \\
\text { responsibilities }\end{array}$ & 3.91 & 1.121 & 3.80 & $\begin{array}{c}1.27 \\
2\end{array}$ \\
\hline $\begin{array}{l}\text { Communication } \\
\text { flaws within } \\
\text { project team }\end{array}$ & 4.11 & 0.959 & 4.00 & 1.16 \\
\hline $\begin{array}{l}\text { Communication } \\
\text { flaws between } \\
\text { project manager } \\
\text { and customer }\end{array}$ & 4.18 & 0.834 & 4.00 & 1.04 \\
\hline $\begin{array}{l}\text { Inadequate } \\
\text { support from } \\
\text { senior } \\
\text { management }\end{array}$ & 4.31 & 1.041 & 4.04 & 1.11 \\
\hline $\begin{array}{l}\text { Inadequate } \\
\text { customer support }\end{array}$ & 4.13 & 1.057 & 4.16 & 1.06 \\
\hline $\begin{array}{l}\text { priority shift } \\
\text { (Changing goals, } \\
\text { business } \\
\text { processes } \\
\text { changed) }\end{array}$ & 3.29 & 1.121 & 3.24 & 1.20 \\
\hline $\begin{array}{l}\text { Time-Boxing } \\
\text { (Insufficient } \\
\text { Time) }\end{array}$ & 4.18 & 0.936 & 4.00 & 1.04 \\
& & & & 9 \\
\hline
\end{tabular}

\begin{tabular}{|l|r|r|r|l|}
\hline $\begin{array}{l}\text { Gold Plating } \\
\text { (Implementing } \\
\text { features without } \\
\text { proper } \\
\text { requirement) }\end{array}$ & 4.09 & 0.996 & 4.04 & 0.903 \\
\hline $\begin{array}{l}\text { Insufficient } \\
\text { supporting tools }\end{array}$ & 2.84 & 1.186 & 2.24 & 1.209 \\
\hline $\begin{array}{l}\text { Lack of technical } \\
\text { expertise }\end{array}$ & 2.13 & 0.991 & 1.91 & 0.996 \\
\hline $\begin{array}{l}\text { Technically } \\
\text { unfeasible } \\
\text { requirement }\end{array}$ & 2.87 & 1.254 & 2.49 & 1.180 \\
\hline $\begin{array}{l}\text { Immeasurable } \\
\text { non functional } \\
\text { requirements }\end{array}$ & 2.73 & 1.074 & 2.33 & 1.087 \\
\hline $\begin{array}{l}\text { Weak access to } \\
\text { customer needs }\end{array}$ & 3.51 & 1.141 & 3.29 & 1.141 \\
\hline $\begin{array}{l}\text { Terminological } \\
\text { problem }\end{array}$ & 2.62 & 1.4351 & 2.69 & 1.520 \\
\hline $\begin{array}{l}\text { Customer } \\
\text { resistance in } \\
\text { signing off } \\
\text { requirement spec }\end{array}$ & 2.22 & 1.126 & 1.98 & 1.097 \\
\hline
\end{tabular}

The variability of the above factors were also assessed by different categories of software firms. There was need to know whether the firms of different size experience the same factors as regards to the RE phase. This was achieved by considering thefollowing hypothesis which was tested to investigate whether the difficulties encountered in the RE phasediffer from the small, medium and large software organisations.

\section{Hypothesis}

Ho: There was no significant difference in the factors affecting the RE phase by the small, medium and large enterprises

H1: There was a significant difference in the factors affecting the RE phase by the small, medium and large enterprises

ANOVA which is parametric test was used as the data was found to be normal ( $p$-value $=0.078$ under Shapiro-Wilk). An index named DI was created to measure the level of difficulty as indicators.

Ho: $\mu$ small $=\mu$ medium $=\mu$ large

H1: $\mu$ small $\neq \mu$ medium $\neq \mu$ large

where $\mu$ small $=$ median value of DI for practitioners from the small enterprise; $\mu$ medium $=$ median value of DI for practitioners from the medium enterprise and $\mu$ large $=$ median value of DI for practitioners from the large enterprise

The ANOVA test revealed that $\mathrm{F}=0.418$ for $\mathrm{p}$-value $=$ $0.662>0.05$ as illustrated in Table 2, indicating that there was no significant difference in the factors identified by the practitioners at the different types of enterprises. The identified factors are common sources of difficulties encountered during development irrespective of sizes of organisation. This provides further evidence that the software 
firms irrespective of size experience similar difficulties for the RE phase.

\begin{tabular}{|l|r|r|r|r|l|}
\hline & $\begin{array}{c}\text { TABLE II: ANOVA FOR THE DI INDEX } \\
\text { Squares }\end{array}$ & df & $\begin{array}{c}\text { Mean } \\
\text { Square }\end{array}$ & F & Sig. \\
\hline Between Groups & 0.052 & 2 & 0.026 & 0.418 & 0.662 \\
\hline Within Groups & 2.322 & 37 & 0.063 & & \\
\hline Total & 2.374 & 39 & & & \\
\hline
\end{tabular}

\section{CONCLUSION \& RECOMMENDATION}

$\mathrm{RE}$ is seen to represent the first leaf of the plant and is seen to play a crucial role in making proper development of the plant i.e. it is a crucial step in working towards successful development of software. RE is a critical domain and the success of development depends on good RE practices. Therefore the RE phase must be carefully planned and its features should not be ignored. Gray areas which stayed over in RE will significantly affect the outcome of projects. Similarly stakeholders are considered to be very important, communication and appropriate support are found to be crucial for the smooth running of the RE phase. Practitioners are increasingly required to interact with the stakeholders [9]. Practitioners' mobility on projects should increased to clear out issues with customers, with development teams as well as with their senior management. [16] rightly said that because stakeholders are situated in their working environment, eliciting their needs directly at work would be advantageous instead of doing so in the sterile atmosphere of a meeting room. Practitioners need to move and clear out issues so that this does not impact on time boxing. [17] mentioned that even when time is tight, still think clear what to develop before developing it. From the study practitioners acknowledged also that it is hazardous to proceed with implementation with unclear requirements. The result of this study provides further direction about the factors affecting the RE phase. The study should be replicated by considering larger sample size with e specificity about the process model used by the software. Further studies could look into how the identified factors vary according to process models used for software development.

\section{REFERENCES}

[1] ISO/IEC/ IEEE Standard 29148:2011(E), Systems and software engineering - Life cycle processes Requirements engineering.

[2] D. Pandey, U. Suman, K. Ramani "Social-Organizational Participation Difficulties in Requirement Engineering Process- A Study", Software Engineering Journal, Bioinfo Publication, Navi Mumbai, 2010.

[3] D. Damian, I. Kwan, S. Marczak "Collaborative software engineering", Springer, 2010.

[4] D. Fernandez and S. Wagner "Naming the Pain in Requirements Engineering: A Design for a Global Family of Surveys and First Results from Germany," Information and Software Technology, vol. 57, 2015, pp. 616-643. https://doi.org/10.1016/j.infsof.2014.05.008

[5] B. Cheng, BHC and J. Atlee "Research Directions in Requirements Engineering. In: Future of Software Engineering", IEEE Computer Society, 2007, pp 285-303
https://doi.org/10.1109/FOSE.2007.17

[6] V. Lamsweerde "Requirements engineering in the year 2000: A research perspective," in Proceedings of the 22nd international conference on Software engineering, 2000, pp. 5-19. https://doi.org/10.1145/337180.337184

[7] B. Nuseibeh. and S. Easterbrook "Requirements Engineering A Roadmap", Proceedings of the Conference on the Future of Software Engineering, 2000, pp. 35-46. https://doi.org/10.1145/336512.336523

[8] C. Pacheco and I. Garcia "A systematic literature review of stakeholder identification methods in requirements elicitation", Journal of Systems and Software, Vol. 85, 2012, pp. 2171-2181 https://doi.org/10.1016/j.jss.2012.04.075

[9] M. Babar, M. Ramzan and S. Ghayyur "Challenges and future trends in software requirements prioritization," in International Conference on Computer Networks and Information Technology (ICCNIT), 2011, pp.319-324.

https://doi.org/10.1109/ICCNIT.2011.6020888

[10] T. Hall, S. Beecham and A. Rainer "Requirements problems in twelve software companies: an empirical analysis", Software IEEE Proceeding, Vol. 149, No. 5, 2002, pp. 153-160 https://doi.org/10.1049/ip-sen:20020694

[11] B, Boehm, P. Papaccio "Understanding and controlling software costs", IEEE Trans Software Eng, Vol. 14, No. 10,1998, pp. 1462-1477 https://doi.org/10.1109/32.6191

[12] M. Geogy and Z. Dharani "A Scrutiny of the Software Requirement Engineering process" Procedia Technology, Vol. 25, 2016, pp. 405410. doi:10.1016/j.protcy.2016.08.125

[13] N. Ganesh and S. Thangasamy "Issues identified in the Software Process due to Barriers found during Eliciting Requirement on Agile Software Projects: Insights from India", International Journal of Computer Applications, 2011, Vol. 16, No.5 https://doi.org/10.5120/2011-2713

[14] V. Pekar, M. Felderer, R. Breu Improvement methods for software requirement specifications: A mapping study. In: Quality of Information and Communications Technology (QUATIC), 9th International Conference on the, IEEE, 2014, pp 242-245 https://doi.org/10.1109/QUATIC.2014.40

[15] M. Condori-Fernandez, M. Daneva, R. Wieringa “A systematic mapping study on empirical evaluation of software requirements specifications techniques", Empirical Software Engineering and Measurement Conference, 2012, pp. 502-505

[16] M. Glinz and R. Wieringa "Stakeholders in requirements engineering", IEEE software, Vol. 28, No. 1, 2007, pp. 18-20 https://doi.org/10.1109/MS.2007.42

[17] H. Lai, R. Peng, D. Sun, F. Shao, Y. Liu and Y. Ni "A systematic review of Re-specific wikis for distributed requirements engineering,"Wuhan University, Wuhan, China, Tech. Rep, Vol. 2012530, 2012.

https://doi.org/10.1109/SKG.2012.11 